\title{
The Algebraic versus the Topological Approach to Additive Representations
}

\author{
Peter Wakker
}

University of Nijmegen

\begin{abstract}
It is proved that, under a nontriviality assumption, an additive function on a Cartesian product of connected topological spaces is continuous, whenever the preference relation, represented by this function, is continuous. The result is used to generalize a theorem of Debreu ((1960). Mathematical methods in the social sciences (pp. 16-26). Stanford: Stanford Univ. Press) on additive representations and to argue that the algebraic approach of KLST to additive conjoint measurement is preferable to the more customary topological approach. Applications to the representation of strength of preference relations and to the characterization of subjective expected utility maximization are given. 1988 Academic Press, Inc.
\end{abstract}

\section{INTRODUCTION AND LiteratURE}

A large number of problems from many fields of science come down to the question of how to aggregate several attributes. The simplest and most common method of aggregation is the additive approach, in which every alternative is evaluated quantitatively with respect to every relevant attribute, and then the obtained values are summed to result in the evaluation of the alternative. Some examples, among many others, are French and Vassiloglou (1986) for the context of examination assessment, Wakker (1984a) for the context of decision making under uncertainty, Tversky (1977) for the measurement of similarity, and Koopmans (1972) for dynamic decision making. Because of the multitude of applications, it is desirable to find conditions by which to test the appropriateness of such additive evaluations, e.g., with preferences, "represented" by the additive evaluations, as primitives. Tests of additivity are described in Adams and Fagot (1959; a variation on the Thomsen condition, see Definition 4.2, is tested), Coombs, Bezembinder and Goode (1967; in their Table 3, triple cancellation, see Definition 4.3, is tested), Tversky (1967), Tversky and Krantz (1969), and Lukas (1987). Falmagne (1976) introduces probabilistic choices in additive conjoint measurement, which are desirable when dealing with error in measurement. Necessary and sufficięnt conditions for additive representations have been obtained in Scott (1964)

Reprint requests should be addressed to Peter Wakker, University of Nijmegen, Department of Mathematical Psychology, P. O. Box 9104, 6500 HE Nijmegen, The Netherlands. The discussion of the empirical implications of the representation theorems of this paper has been added on the recommendation of a referee and the editor. Their comments also helped improve the clarity of exposition. 
and Tversky (1964; see also Krantz, Luce, Suppes, and Tversky (KLST), 1971, Sect. 9.2) for the case of finite structures. Unfortunately, these conditions in fact constitute an infinite sequence of axioms, so that there is no straightforward way to test them. For an algorithm to test additive representability, see for instance Roskam (1987). Also, for infinite models necessary and sufficient conditions have been obtained in Jaffray $(1974 a, b)$. These are still more complicated than those for the finite models.

For this reason approaches other than those which gave the necessary and sufficient conditions are usually used. These other approaches use simplifying nonnecessary conditions. The most common nonnecessary conditions are of a topological nature, requiring continuity. The major advance in the use of this approach was the result of Debreu (1960, Theorem 3). Debreu required continuity with respect to a "connected" and "separable" topology. (For elementary topological definitions, see Kelley (1955).) By themselves these topological assumptions are merely "technical." They have no empirical implications and cannot be verified or falsified by observations. Technical assumptions, while not very satisfactory, are not very bothersome either. They merely serve to make mathematical machinery work smoothly. They are void of empirical meaning, and so do not entail obscurity.

However, when one requires a list of conditions, then one should not judge each condition separately, but one should judge the conditions in combination. We give an example (Example 7.3) where, paradoxically, each individual condition involved is not falsified by the observations, but the combination of the conditions is falsified. As it turns out in this example, continuity, in the presence of other conditions, may have empirical meaning. It is very bothersome that usually the exact empirical meaning of simplifying nonnecessary conditions such as continuity is unclear.

An alternative approach has been established, and initiated, by KLST. They also use nonnecessary simplifying assumptions, but these are of an algebraic, instead of a topological, nature. Instead of continuity with respect to a connected (separable) topology, KLST use a solvability axiom (in which we omit the term "restricted") and an Archimedean axiom. There also is obscurity about the empirical content of these conditions, in the presence of other conditions, as is again illustrated by Example 7.3. Still, this obscurity is somewhat less than that of continuity, as at least the Archimedean axiom is purely technical (we will get back to this near the end of Section 7).

Whereas the derivations in the topological approach either, as in Debreu (1960), use results from web theory or, as in Gorman (1968), use results from the theory of functional equations, the derivations in the algebraic approach are based upon algebraic results, such as a variation of the Lemma of Hölder in KLST. KLST, in Theorem 6.14, showed that whenever the topological approach of Debreu, assuming continuity with respect to a connected (and separable) product topology, can be applied, then the solvability and Archimedean axiom of KLST are also satisfied, so that also the algebraic approach can be applied. KLST also give 
examples where the algebraic approach, but not the topological approach, can be applied (see Examples 7.1 and 7.2 in this paper). This is one reason for considering the algebraic approach to be more general. Furthermore, KLST show that, even without the requirement of topological separability, the algebraic approach can still be applied. This is a second reason for considering the algebraic approach to be more general. However, contrary to what is suggested in KLST above Theorem 6.14 (compare our Section 6), these two reasons are not yet sufficient for concluding that their results are more general than those of Debreu (1960) because Debreu also derived continuity of his representation; in KLST this is not done. The present paper aims to supply this last detail to the work of KLST. So we establish, in our main result, Theorem 3.1, continuity of additive representations, on the basis of which it can be decided definitively that the algebraic approach has led to more general results than the topological approach. With this established, in Sections 4 to 6 we obtain generalizations of previously published results using the topological approach.

At first sight it may appear that the result of Theorem 3.1 is trivial. To the best of our knowledge, it is not. First let us note that, contrary to what is sometimes thought, continuity of a binary relation does not automatically imply continuity of any representing function. Any (strictly) increasing, noncontinuous function on the reals shows this. However, Debreu (1964) showed that there always exists a continuous representing function, if the binary relation is continuous. But then, there still is no reason to suppose that the additive representing function considered in Theorem 3.1 will be such a continuous representing function. Let us also point out here that the Cartesian product structure is so dominant in the proof of Theorem 3.1 that, contrary to what might be expected, the derivations of continuity results for "non-Cartcsian products," as in Debrcu (1964), Bowen (1968), and Jaffray (1975), have not been of direct use in this proof. Jaffray (1974a, Sects. V.7.2 and V.8) obtained a characterization of continuity of additive representations in terms of an $L(\cdot, \cdot, \cdot, \cdot)$ function, which measures more or less proportions of differences of utilities. Using this, Jaffray (1974a, Sect. VI.2.2) supplied continuity for the derivation of the additive representations of Luce (1966), for the case of two coordinates. Jaffray (1974a, p. 97) already dispensed with topological separability when he dealt with the result of Debreu (1960).

\section{Preliminaries}

Throughout the sequel we assume:

Assumption 2.1. $\mathscr{C}_{1}, \ldots, \mathscr{C}_{n}$ are connected topological spaces, with $n$ an arbitrary fixed natural number. The Cartesian product $X_{i=1}^{n} \mathscr{C}_{i}$ is endowed with the product topology. By $\geqslant$ a binary relation on $X_{i=1}^{n} \mathscr{C}_{i}$ is denoted.

Let us recall that a topological space $\mathscr{C}$ is connected if $\phi$ and $\mathscr{C}$ are the only subsets of $\mathscr{C}$ which are both open and closed. Examples of connected topological 
spaces are $\mathbb{R}_{+}^{m}$, or $\mathbb{R}$. For an element $x$ of $X_{i=1}^{n} \mathscr{C}_{i}, x_{j}$ is the $j$ th coordinate of $x$. Some standard notations for $\succcurlyeq$ are the following.

We write $x \leqslant y$ if $y \geqslant x ; x \approx y$ if $x \geqslant y$ and $y \geqslant x ; x>y$ if $x \geqslant y$ and not $y \geqslant x$; $x<y$ if $y \succ x$. We call $\geqslant$ a weak order if it is both complete $(x \geqslant y$ or $y \geqslant x$, for all $\left.x, y \in X_{i=1}^{n} \mathscr{C}_{i}\right)$ and transitive. Then $\approx$ is an equivalence relation. We call $\geqslant$ continuous if $\left\{x \in X_{i=1}^{n} \mathscr{C}_{i}: x \geqslant y\right\}$ and $\left\{x \in X_{i=1}^{n} \mathscr{C}_{i}: x \preccurlyeq y\right\}$ are closed for all $y \in X_{i=1}^{n} \mathscr{C}_{i}$.

Notation 2.2. For $x \in X_{k=1}^{n} \mathscr{C}_{k}, 1 \leqslant i \leqslant n, 1 \leqslant j \leqslant n, v_{i} \in \mathscr{C}_{i}, w_{j} \in \mathscr{C}_{j}, x_{-i} v_{i}$ is ( $x$ with $x_{i}$ replaced by $\left.v_{i}\right)$, and for $j \neq i,\left(x_{-i, j} v_{i}, w_{j}\right)$ is $\left(x\right.$ with $x_{i}$ replaced by $v_{i}$, and $x_{j}$ replaced by $w_{j}$ ).

Definition 2.3. Coordinate (or index) $i$ is inessential if $x_{-i} v_{i} \approx x$ for all $x \in X_{j=1}^{n} \mathscr{C}_{j}, v_{i} \in \mathscr{C}_{i}$. The opposite of inessential is essential.

The following property is known by many names, such as (preferential) independence, additivity, strong (strict) separability, and the sure-thing principle. The term below abbreviates "independent of equal coordinates."

DefinItION 2.4. We say $\geqslant$ is coordinate independent $(C I)$ if, for all $x$, $y \in X_{j=1}^{n} \mathscr{C}_{j}, 1 \leqslant i \leqslant n, v_{i}, w_{i} \in \mathscr{C}_{i}$,

$$
x_{-i} v_{i} \succcurlyeq y_{-i} v_{i} \Leftrightarrow x_{-i} w_{i} \succcurlyeq y_{-i} w_{i} .
$$

If $\geqslant$ is $\mathbf{C I}$, then the following notation is useful:

DefinIIION 2.5. The binary relation $\succcurlyeq_{i}$ on $\mathscr{C}_{i}$ is defined by $v_{i} \succcurlyeq_{i} w_{i}$ if therc exists an $x \in X_{j=1}^{n} \mathscr{C}_{j}$ such that $x_{-i} v_{i} \geqslant x_{-i} w_{i}$.

The binary relations $\succ_{i}, \approx_{i}, \preccurlyeq_{i}, \prec_{i}$ are derived from $\succcurlyeq_{i}$ in the same way as that was done without the index $i$.

LeMma 2.6. Let $\geqslant$ be a $C I$ weak order on $X_{j=1}^{n} \mathscr{C}_{j}$. Then we have, for every $1 \leqslant i \leqslant n:\left[v_{i} \succcurlyeq_{i} w_{i} \Leftrightarrow x_{-i} v_{i} \geqslant x_{-i} w_{i}\right]$ for all $x ; \succcurlyeq_{i}$ is a weak order; $\preccurlyeq_{i}, \prec_{i}, \succ_{i}, \approx_{i}$ can be derived from $\preccurlyeq,\left\langle, \succ, \approx\right.$ in the same way as $\geqslant_{i}$ is derived from $\geqslant$; if $\geqslant$ is continuous, then so is $\succcurlyeq_{i}$.

Proof. All these results are elementary; some of them are given as exercises in KLST (Section 6.1.4). The last statement can be inferred from the proof of Theorem 6.14 in KLST.

Definition 2.7. We say $\geqslant$ satisfies restricted solvability if, for all $x_{-i} a_{i} \geqslant y \geqslant$ $x_{-i} c_{i}$, there exists a $b_{i}$ such that $x_{-i} b_{i} \approx y$.

The following result is derived in the first lines of the proof of Theorem 6.14 in KLST. 
LEMMA 2.8. If $\geqslant$ is a continuous $C l$ weak order, then it satisfies restricted solvability.

Next we give some standard terminologies for a function $V: X_{i=1}^{n} \mathscr{C}_{i} \rightarrow \mathbb{R}$. $V$ represents $\succcurlyeq$ if, for all $x, y \in X_{i=1}^{n} \mathscr{C}_{i},[x \geqslant y \Leftrightarrow V(x) \geqslant V(y)] . V$ is additive if there exist $V_{j}: \mathscr{C}_{j} \rightarrow \mathbb{R}, j=1, \ldots, n$, such that $V(x)=\sum_{j=1}^{n} V_{j}\left(x_{j}\right)$ for all $x$. The following is verified straightforwardly:

LEMMA 2.9. If $\geqslant$ can be represented by an additive function, then $\succcurlyeq$ is $C I$.

\section{The Main Theorem}

The following result is central to this paper.

THEOREM 3.1. Let $V: X_{i=1}^{n} \mathscr{C}_{i} \rightarrow \mathbb{R}$. Let the following five conditions hold:

(i) $V$ is additive.

(ii) $\mathscr{C}_{1}, \ldots, \mathscr{C}_{n}$ are connected topological spaces.

(iii) $X_{i-1}^{n} \mathscr{C}_{i}$ is endowed with the product topology.

(iv) The binary relation $\geqslant$ on $X_{i=1}^{n} \mathscr{C}_{i}$, represented by $V$, is continuous.

(v) At least two coordinates are essential.

Then this additive function $V$ is also continuous.

Proof. For $j=1, \ldots, n$, let $V_{j}: \mathscr{C}_{j} \rightarrow \mathbb{R}$ be such that $V(x)=\sum_{j=1}^{n} V_{j}\left(x_{j}\right)$ for all $x$. It is sufficient to prove that every $V_{j}$ is continuous. So we suppose that $V_{1}$ is not continuous, and derive contradiction.

For some $\mu \in \mathbb{R}$, either $V_{1}^{-1}(]-\infty, \mu[)$ is not open or $V_{1}^{-1}(] \mu, \infty[)$ is not open; say the latter. Then certainly

$$
\phi \neq V_{1}^{-1}(] \mu, \infty[) \neq \mathscr{C}_{1} .
$$

Also there can be no sequence $\left(V_{1}\left(x_{1}^{k}\right)\right)_{k=1}^{\infty}$ in $] \mu, \infty$ [, converging to $\mu$, because then, $V_{1}$ representing $\succcurlyeq_{1}, V_{1}^{-1}(] \mu, \infty[)$ would equal $U_{k}\left\{z_{1}: z_{1}>_{1} x_{1}^{k}\right\}$, so that by Lemmas 2.9 and $2.6\left(\succcurlyeq_{i}\right.$ is a continuous weak order) openness of $V_{1}^{-1}(] \mu, \infty[)$ would result. We conclude that

$$
\left.\inf V_{1}\left(\mathscr{C}_{1}\right) \cap\right] \mu, \infty[=: v \text { satisfies } v>\mu .
$$

Next we shall derive

$$
0<V_{j}\left(x_{j}\right)-V_{j}\left(y_{j}\right)<v-\mu \quad \text { for no } j \neq 1, x_{j}, y_{j} .
$$

If, to the contrary, $j \neq 1$ and $0<V_{j}\left(x_{j}\right)-V_{j}\left(y_{j}\right)<v-\mu$, then, with $z \in X_{i=1}^{n} \mathscr{C}_{i}$ 
arbitrarily fixed, with $a_{1} \in \mathscr{C}_{1}$ such that $V_{j}\left(x_{j}\right)-V_{j}\left(y_{j}\right)>V_{1}\left(a_{1}\right)-v \geqslant 0$, and with $c_{1} \in \mathscr{C}_{1}$ such that $V_{1}\left(c_{1}\right) \leqslant \mu$ (by (3.1) such a $c_{1}$ exists), we get for these $a_{1}, c_{1}$

$$
\left(z_{-1, j} c_{1}, x_{j}\right)<\left(z_{-1, j} a_{1}, y_{j}\right)<\left(z_{-1, j} a_{1}, x_{j}\right),
$$

by substitution of inequalities in terms of the $V_{j}$ s. By restricted solvability (Lemma 2.8), there exists a $b_{1}$ such that $\left(z_{-1, j} b_{1}, x_{j}\right) \approx\left(z_{-1, j} a_{1}, y_{j}\right)$. This would now imply $v>V_{1}\left(b_{1}\right)>\mu$, in contradiction with the definition of $v$. Apparently (3.3) must hold.

There is a $j \neq 1$ which is essential, from which it follows that $V_{j}$ is not constant. Say $V_{j}\left(x_{j}\right)>V_{j}\left(y_{j}\right)$. As a consequence of (3.3), a $z_{j}$ must exist such that $V_{j}\left(x_{j}\right)>$ $V_{j}\left(z_{j}\right)$, and $V_{j}\left(x_{j}\right)>V_{j}\left(v_{j}\right)>V_{j}\left(z_{j}\right)$ for no $v_{j} \in \mathscr{C}_{j}$. This finally gives $\left\{v_{j} \in \mathscr{C}_{j}\right.$ : $\left.v_{j} \preccurlyeq_{j} z_{j}\right\}=\left\{v_{j} \in \mathscr{C}_{j}: v_{j} \prec_{j} x_{j}\right\}$, where this latter set contains $z_{j}$ so it is nonempty, does not contain $x_{j}$ so it does not equal $\mathscr{C}_{j}$, and is both open and closed by continuity of the weak order $\succcurlyeq_{i}$. Contradiction with connectedness of $\mathscr{C}_{j}$ follows.

\section{Applications to Additive Representations}

The first theorem of this section extends Theorem 3 of Debreu (1960) and Theorem 6.14 of KLST.

THEOREM 4.1. Let $\mathscr{C}_{1}, \ldots, \mathscr{C}_{n}$ be connected topological spaces; let $X_{i=1}^{n} \mathscr{C}_{i}$ be endowed with the product topology. Let $\geqslant$ be a binary relation on $X_{i=1}^{n} \mathscr{C}_{i}$, with at least three coordinates essential. Then the following two statements are equivalent:

(i) There exists a continuous additive representation for $\geqslant$.

(ii) The binary relation $\geqslant$ is a continuous coordinate independent weak order.

Proof. The implication (i) $\Rightarrow$ (ii) is straightforward, so we assume (ii), and derive (i). By Theorem 6.14 of KLST, there exists an additive representation. By our Theorem 3.1 this representation is continuous. So (i) holds.

Next the results of KLST for the case of exactly two essential coordinates are adapted to the topological approach. For notational simplicity we shall assume that $n=2$ and that both coordinates are essential. Inessential coordinates do not affect binary relations which are weak orders, and a function which represents $\geqslant$ is independent of the inessential coordinates. Hence inessential coordinates may just as well be suppressed from notation.

Definition 4.2. Let $n=2$. We say that $\geqslant$ satisfies the Thomsen condition if, for all $a_{1}, b_{1}, c_{1} \in \mathscr{C}_{1}$ and $s_{2}, t_{2}, v_{2} \in \mathscr{C}_{2},\left(a_{1}, t_{2}\right) \approx\left(b_{1}, s_{2}\right)$ and $\left(b_{1}, v_{2}\right) \approx\left(c_{1}, t_{2}\right)$ together imply $\left(a_{1}, v_{2}\right) \approx\left(c_{1}, s_{2}\right)$. 
DEFINITION 4.3. Let $n=2$. We say that $\geqslant$ satisfies triple cancellation if, for all $a_{1}, b_{1}, c_{1}, d_{1} \in \mathscr{C}_{1}$ and $s_{2}, t_{2}, v_{2}, w_{2} \in \mathscr{C}_{2},\left(a_{1}, s_{2}\right) \preccurlyeq\left(b_{1}, t_{2}\right)$ and $\left(c_{1}, s_{2}\right) \geqslant\left(d_{1}, t_{2}\right)$ and $\left(a_{1}, v_{2}\right) \geqslant\left(b_{1}, w_{2}\right)$ together imply $\left(c_{1}, v_{2}\right) \geqslant\left(d_{1}, w_{2}\right)$.

THEOREM 4.4. Let $\mathscr{C}_{1}, \mathscr{C}_{2}$ be two connected topological spaces; let $\mathscr{C}_{1} \times \mathscr{C}_{2}$ be endowed with the product topology. Let $\geqslant$ be a binary relation on $\mathscr{C}_{1} \times \mathscr{C}_{2}$, with both coordinates essential. Then the following three statements are equivalent:

(i) There exists a continuous additive representation for $\succcurlyeq$.

(ii) The binary relation $\geqslant$ is a continuous coordinate independent weak order that satisfies the Thomsen condition.

(iii) The binary relation $\geqslant i s$ a continuous weak order that satisfies triple cancellation.

Proof. The implications (i) $\Rightarrow$ (ii) and (i) $\Rightarrow$ (iii) are straightforward. To derive (ii) $\Rightarrow$ (i) [respectively (iii) $\Rightarrow($ i)], we assume (ii) [respectively (iii)]. In the proof of Theorem 6.14 in KLST, it is shown that continuity of $\geqslant$ w.r.t. a connected product topology implies restricted solvability and the Archimedean axiom/property there. Although this is done for a case with three or more essential coordinates, the reasoning literally applies to our case, with two essential coordinates. Hence, by Theorem 6.2 [respectively the indications at the end of Section 6.2.4] of KLST, an additive representation exists for $\geqslant$, this being a weak order which satisfies restricted solvability, the Archimedean axiom, and further $\mathrm{CI}$ and the Thomsen condition [respectively further triple cancellation]. By our Theorem 3.1, the additive representation is continuous.

Gorman (1968, Sect. 5, corollary of Theorem 2) strengthened Theorem 3 of Debreu (1960) (i.e., our Theorem 4.1 restricted to (topologically) separable $\mathscr{C}_{j}$ 's) by showing that the coordinate independence condition can be weakened. Gorman did this for separable arc-connected spaces $\mathscr{C}_{j}$. The question whether the above-mentioned, and other, results of Gorman (1968) also hold for connected (instead of arcconnected) separable spaces initiated a discussion in the literature. Murphy (1981) refers to Gorman (1971) and Vind (1971), and Sertel (1972) and Vind (1974); furthermore, Murphy gives an elementary proof to show that Gorman's results do indeed hold for connected separable spaces. The results of this section suggcst a topic for future research: Do (some of) Gorman's results also hold for connected topological spaces which are not separable?

We have not paid attention to uniqueness results. They are standard, and the additive representations $V$ that we derived are all cardinal, i.e., can be replaced by a function $V^{*}$ if and only if a real $\tau$ and a positive $\sigma$ exist such that $V^{*}=\tau+\sigma V$. This is proved in KLST. 
5. An Application to the Representation of Strength of Preference

In this section we assume that $n=2, \mathscr{C}_{1}=\mathscr{C}_{2}=\mathscr{C}$. Greek characters denote elements of $C$. We interpret $\geqslant$ on $\mathscr{C}^{2}$ as a "strength of preference relation"; i.e., $(\alpha, \beta) \geqslant(\gamma, \delta)$ means that $\alpha$ is preferred to $\beta$ at least as intensively as $\gamma$ to $\delta$. The following two conditions of KLST are central to the characterization of a strength of preference relation through utility differences.

Definition 5.1. The binary relation $\geqslant$ on $\mathscr{C}^{2}$ satisfies the reversal condition if, for all $\alpha, \beta, \gamma, \delta \in \mathscr{C},[(\alpha, \beta) \geqslant(\gamma, \delta) \Rightarrow(\delta, \gamma) \geqslant(\beta, \alpha)]$.

DEFINITION 5.2. The binary relation $\succcurlyeq$ on $\mathscr{C}^{2}$ satisfies the concatenation condition if, for all $\alpha, \beta, \gamma, \alpha^{\prime}, \beta^{\prime}, \gamma^{\prime} \in \mathscr{C},\left[(\alpha, \beta) \geqslant\left(\alpha^{\prime}, \beta^{\prime}\right)\right.$ and $\left.(\beta, \gamma) \geqslant\left(\beta^{\prime}, \gamma^{\prime}\right)\right] \Rightarrow$ $\left[(\alpha, \gamma) \geqslant\left(\alpha^{\prime}, \gamma^{\prime}\right)\right]$.

With these we get:

TheOREM 5.3. Let $\mathscr{C}$ be a connected topological space, and let $\geqslant$ be a binary relation on $\mathscr{C}^{2}$. The following two statements are equivalent:

(i) There exists a continuous function $U: \mathscr{C} \rightarrow \mathbb{R}$ such that $\geqslant$ is represented by the function $(\alpha, \beta) \mapsto U(\alpha)-U(\beta)$.

(ii) The binary relation $\geqslant$ is a continuous weak order, that satisfies the reversal and concatenation conditions.

Proof. The implication (i) $\Rightarrow$ (ii) is straightforward. So we assume (ii) and derive (i). In Definition 4.3 in KLST, conditions $1(\geqslant$ is a weak order), 2 (the reversal condition), and 3 (the concatenation condition) are directly seen to hold; conditions 4 and 5 are closely related to restricted solvability and the Archimedean axiom and can be derived from continuity of $\geqslant$, analogously to the proof of Theorem 6.14 in KLST. We do not elaborate on this. Once these five conditions are verified, by Theorem 4.2 of KLST there exists a function $U: \mathscr{C} \rightarrow \mathbb{R}$ which, with the exception of continuity, satisfies all of statement (i).

The continuity of $U$ remains to be derived. If coordinate 1 is inessential, then $U$ must be constant, and so continuous. If coordinate 1 is essential, then so is coordinate 2 , by the reversal condition. By Theorem 3.1, the function which assigns $U(\alpha)-U(\beta)$ to every $(\alpha, \beta) \in \mathscr{C}^{2}$ is continuous. So $U$ is continuous.

The above theorem shows how Theorem 4.2 of KLST can be used to generalize earlier results in the literature for topological contexts; see the beginning of Sect. 4.4 in KLST for early references. In a more recent study by Shapley (1975; given in Shubik, 1982, Appendix 3 ), for the case that $\mathscr{C}$ is a convex subset of $\mathbb{R}$, a characterization of (i) in the above theorem (with $U$ strictly increasing) is given, in which the reversal and concatenation conditions are replaced by monotonicity conditions (implying CI) and a "crossover axiom" $[(\alpha, \beta) \approx(\gamma, \delta) \Leftrightarrow(\alpha, \gamma) \approx(\beta, \delta)]$. Shapley's 
work turned out to be a generalization of Alt (1936). We conjecture that Shapley's result can also be extended to any connected topological space $\mathscr{C}$.

In Sect. 6 of Wakker (1988) an alternative way to characterize (i) in Theorem 5.3 is indicated. The main condition used there is equivalence cardinal coordinate independence, obtained from Definition 6.1 (introduced in the next section) by replacing $\preccurlyeq$, and all $\succcurlyeq$ 's, by $\approx$.

Uniqueness results concerning the function $U$ in (i) of Theorem 5.3 (this $U$ is cardinal) have been discussed extensively since this played a role in the cardinal versus ordinal utility discussion around 1930. Basu (1982) provides references on the history of this topic. A recent study of this discussion can be found in Cooter and Rappoport (1984; see also Little, 1985; Cooter and Rappoport, 1985). Basu extends the uniqueness result for the function $U$ above by showing that continuity of $U$ is not necessary: connectedness of $U(\mathscr{C})$ is already sufficient. The approach of KLST shows a way to further extend this: the only nonnecessary condition (4), some sort of restricted solvability condition, used in the derivation of a cardinal utility function $U$ in Theorem 4.2 of KLST, is implied by connectedness of $U(\mathscr{C})$ and is essentially weaker than connectedness of $U(\mathscr{C})$, as can be inferred from the lines above Definition 6.14 in KLST (let $\mathscr{C}$ be the set of rational numbers, $U$ identity, etc.).

\section{An Application to Decision Making Under UnCertainty}

We deferred until the end a further application of Theorem 3.1: it supplements an omission in a proof published earlier by the author. Let us assume that $\mathscr{C}_{1}=\cdots=\mathscr{C}_{n}=\mathscr{C}$; again Greek characters denote elements of $\mathscr{C}$. There is a set of $n$ states of nature, of which exactly one is true; the others are untrue. An element $x=\left(x_{1}, \ldots, x_{n}\right)$ of $\mathscr{C}^{n}$ is an "act," yielding "consequence" $x_{j} \in \mathscr{C}_{j}$ if the $j$ th state of nature is the true one. The binary relation $\geqslant$ is the "preference relation" of a decision maker, who is uncertain about which state of nature is the true one. Elucidation of the following condition is given in Wakker (1984a). A motivation for the chosen term can be inferred from the italicized part of the proof sketch of Theorem 6.2.

Definition 6.1. The binary relation $\geqslant$ on $\mathscr{C}^{n}$ satisfies cardinal coordinate independence $(\mathrm{CCI})$ if, for all $x, y, v, w \in \mathscr{C}^{n} ; \alpha, \beta, \gamma, \delta \in \mathscr{C} ; 1 \leqslant j \leqslant n$; and $1 \leqslant i \leqslant n$ with $i$ essential, the three preferences

$$
x_{-i} \alpha \preccurlyeq y_{-i} \beta, \quad v_{-j} \alpha \geqslant w_{-j} \beta, \quad x_{-i} \gamma \geqslant y_{-i} \delta
$$

together imply the fourth preference $v_{-j} \gamma \geqslant w_{-j} \delta$.

The following theorem characterizes "subjective expected utility maximization" with a continuous utility function. It was published before in Wakker (1984a) for the case where $\mathscr{C}$ is an open interval, and in Wakker (1986, Theorem 5.1) in its 
present form. Unfortunately, in the proof of the latter there was an omission in the derivation of continuity of the obtained "utility function" $U$. The author was mistaken in his belief that in (the proof of) Theorem 6.14 in KLST continuity of the representing function was also derived, as it was in Debreu (1960, Theorem 3). Out of this omission the present paper has evolved. Continuity of the function $U$ below only now follows, after the derivation of Theorem 3.1, with the substitutions $V_{i}:=p_{i} U$. So we now have:

THEOREM 6.2. Let $\mathscr{C}$ be a connected topological space, and $\succcurlyeq$ a binary relation on $\mathscr{C}^{n}$ with at least two coordinates essential. Then the following two statements are equivalent:

(i) There exist nonnegative $p_{1}, \ldots, p_{n}$, summing to one, and a continuous function $U: \mathscr{C} \rightarrow \mathbb{R}$, such that, for all $\left(x_{1}, \ldots, x_{n}\right)$ and $\left(y_{1}, \ldots, y_{n}\right) \in \mathscr{C}^{n}$,

$$
\left(x_{1}, \ldots, x_{n}\right) \geqslant\left(y_{1}, \ldots, y_{n}\right) \Leftrightarrow \sum_{j=1}^{n} p_{j} U\left(x_{j}\right) \geqslant \sum_{j=1}^{n} p_{j} U\left(y_{j}\right)
$$

(ii) The binary relation $\succcurlyeq$ is a continuous weak order which satisfies cardinal coordinate independence.

Proof Sketch of (ii) $\Rightarrow$ (i). The CCI condition can be seen to imply $C I$, and triple cancellation for $n=2$. Hence by Section 4 an additive representation $V$, say $V: x \mapsto \sum V_{j}\left(x_{j}\right)$, exists, with all $V_{j}$ continuous. Now CCI implies, roughly, that $V$ orders ("first-order") differences the same way across states (coordinates), by the $V_{j}^{\prime}$ 's. As Basu (1982) showed, this implies the $V_{j}^{\prime}$ 's to be in the same "cardinal class," the $V_{j}^{\prime}$ 's being continuous functions on connected domains. Giving the $V_{j}^{\prime}$ 's a common zero leads to statement (i).

The adaptation of the above theorem to arbitrary, possibly infinite, state spaces has been given in Wakker (1984b). Grodal (1978, Theorem 3) gave an alternative characterization of subjective expected utility maximization with continuous utility in a slightly different model. The main difference from our result is that, instead of cardinal coordinate independence, she uses a condition in terms of a mean groupoid operation which is derived from the preference relation, using continuity.

Again uniqueness results are standard, the function $U$ above is cardinal, and the "subjective probabilities" $p_{1}, \ldots, p_{n}$ are uniquely determined.

\section{EXAMPLES AND DisCUSSION}

A first contribution of Theorem 3.1 is one of a mathematical nature. It supplies the last tool needed to show that the topological result of Debreu (1960) can fully be obtained as a corollary of the algebraic approach, and that this alternative algebraic approach in fact leads to a more general result than the method of 
Debreu (1960). Some generalizations of existing results that can be derived by this method have been given in previous sections.

Let us now prepare for a discussion of the significance of Theorem 3.1 for the (non)testability of axioms. Analogous remarks apply to the results as derived from this theorem in previous sections. First we compare the algebraic approach and the topological approach. So we make a comparison between, on the one hand, the combination of solvability and the Archimedean axiom, and, on the other hand, continuity with respect to a connected topology. We give two examples of KLST to show that the algebraic approach is applicable to more cases than the topological approach. In the examples the algebraic conditions are satisfied, and the approach of KLST can be applied. The topological conditions of continuity with respect to a connected topology cannot be satisfied in these examples, for instance, because the examples concern countable spaces, whereas the continuous image of a connected topological space, if not degenerate, is an interval, and so contains an uncountable number of points.

EXAMPLE 7.1. Let $X=\{1,2,3\}^{3}$. Let $\left(x_{1}, x_{2}, x_{3}\right) \geqslant\left(y_{1}, y_{2}, y_{3}\right)$ if and only if $x_{1}+x_{2}+x_{3} \geqslant y_{1}+y_{2}+y_{3}$. Obviously $\left[V_{1}=V_{2}=V_{3}=\right.$ identity $]$ yields an additive representation for $\geqslant$. This is an example of an "equally spaced" structure as indicated in KLST.

EXAMPLE 7.2. Let $X=\mathbb{Q}^{3}$. Again let $\left(x_{1}, x_{2}, x_{3}\right) \geqslant\left(y_{1}, y_{2}, y_{3}\right)$ if and only if $x_{1}+x_{2}+x_{3} \geqslant y_{1}+y_{2}+y_{3}$. Again $\left[V_{1}=V_{2}=V_{3}=\right.$ identity $]$ yields an additive representation for $\succcurlyeq$. This example was indicated by KLST (1971, p. 302).

Let us next point out a difficulty in the (non)testability of continuity and solvability. This was already explicated in KLST (1971, Sect. 9.1).

EXAmPLE 7.3. Suppose a person has a preference relation $\geqslant$ on $\mathbb{R}^{3}$, for the definition of which we first introduce functions $V_{1}, V_{2}, V_{3}: \mathbb{R} \rightarrow \mathbb{R}$. Let $V_{1}(\mu)=1$ for all $\mu>0, V_{1}(0)=0, V_{1}(\mu)=-1$ for all $\mu<0$. Let $V_{2}=V_{1}$. Let $V_{3}=5 \times V_{1}$. Suppose that in fact $\left(x_{1}, x_{2}, x_{3}\right) \geqslant\left(y_{1}, y_{2}, y_{3}\right)$ if and only if $\sum_{j=1}^{n} V_{j}\left(x_{j}\right) \geqslant \sum_{j=1}^{n} V_{j}\left(y_{j}\right)$, with one exception. If $V_{1}\left(x_{1}\right)=V_{2}\left(y_{2}\right)=1, V_{2}\left(x_{2}\right)=V_{1}\left(y_{1}\right)=0$, and $V_{3}\left(x_{3}\right)=V_{3}\left(y_{3}\right)$, then not $\left(x_{1}, x_{2}, x_{3}\right) \approx\left(y_{1}, y_{2}, y_{3}\right)$, but $\left(x_{1}, x_{2}, x_{3}\right)>\left(y_{1}, y_{2}, y_{3}\right)$. Obviously the preference relation is a weak order, and all three coordinates arc essential. It is tedious, but straightforward, to check that the preference relation satisfies coordinate independence. It does not satisfy restricted solvability, as follows from $(1,0,1) \succ(0,0,1) \succ(1,0,0)$ and the nonexistence of $\mu$ such that $(1,0, \mu) \approx(0,0,1)$. Hence a fortiori it is not continuous. Now suppose a scientist wants to find out about this preference relation, in particular if it is additively representable. The scientist observes the following preferences: $(1,1,1) \succ(1,0,1) \succ(0,1,1) \succ$ $(1,-1,1) \approx(0,0,1) \approx(-1,1,1) \succ(-1,0,1) \approx(0,-1,1) \succ(-1,-1,1) \succ$ $(1,1,0)>(1,0,0) \succ(0,1,0)>(1,-1,0) \approx(0,0,0) \approx(-1,1,0)>(-1,0,0)$ 
$\approx(0,-1,0) \succ(-1,-1,0)>(1,1,-1)>(1,0,-1)>(0,1,-1)>$ $(1,-1,-1) \approx(0,0,-1) \approx(-1,1,-1)>(-1,0,-1) \approx(0,-1,-1) \succ$ $(-1,-1,-1)$. These preferences indeed reveal that all coordinates are essential, and obviously they do not reveal a violation of coordinate independence, transitivity, or completeness. But neither can they reveal a violation of continuity or solvability. It is straightforward, but tedious, to construct a preference relation which satisfies continuity and hence solvability and which agrees with all preferences as observed by the scientist. Suppose that the scientist would not be aware of the fact that continuity and solvability, while by themselves without empirical meaning, do add empirical meaning to the other axioms. Then he might think, on the basis of Theorem 4.1, that an additive representation exists for the observed preferences, or at least that his observations do not falsify additive representability. The scientist would be mistaken in this. Whereas no single condition of statement (ii) in Theorem 4.1 can be falsified, their combination can be, paradoxical as this may seem.

To see this, suppose $W_{1}, W_{2}, W_{3}$ would yield an additive representation. Then the preferences $(-1,0,0) \approx(0,-1,0)$ and $(1,-1,0) \approx(-1,1,0)$ would imply the equalities $W_{1}(-1)+W_{2}(0)=W_{1}(0)+W_{2}(-1)$ and $W_{1}(1)+W_{2}(-1)=$ $W_{1}(-1)+W_{2}(1)$. Summing left sides, and summing right sides, and cancelling terms $W_{1}(-1)$ and $W_{2}(-1)$ yield $W_{1}(1)+W_{2}(0)=W_{1}(0)+W_{2}(1)$. This should imply $(1,0,0) \approx(0,1,0)$, in contradiction with the observed $(1,0,0)>(0,1,0)$. The finite number of observations does already falsify additive representability. If the scientist supposes that the preference relation is a coordinate independent weak order, then from a finite number of observations he can, on the basis of Theorem 4.1, conclude that the preference relation does not satisfy solvability, and therefore not continuity.

Let us emphasize that, of course, the exact empirical content which continuity and/or solvability add to other conditions depends on what these other conditions are. The statements in this paper all apply to the case of statements (ii)/(iii) in Theorems 4.1, 4.4, 5.3, 6.2. An alternative approach, very interesting in relation to the present discussion, is given by Fuhrken and Richter (1988). Instead of the CI condition in statement (ii) in Theorem 4.1, or analogous conditions in statements (ii)/(iii) in Theorem 4.4, they require conditions of Scott (1964), which is equivalent to requiring all "cancellation axioms" of KLST, rather than just one or two as is done in most other approaches, including that of our paper. These cancellation axioms are necessary and sufficient for additive representability in the case of finite structures. Furthermore, Fuhrken and Richter (1988) use topological connectedness, already dispense with topological separability, and use a "sectional continuity" condition which is somewhat weaker than continuity. By requiring all cancellation axioms, Fuhrken and Richter (1988) succeed in making the continuity condition purely technical. A problem with the cancellation axioms is that they form an infinite list of axioms (Adams, Fagot, and Robinson (1970) refer to Titiev (1969) for this), so that thcir empirical verification remains problematic. Adams, Fagot, and Robinson (1970, p. 406, lines 12-13) consider a case where there are more nonnecessary conditions in addition to continuity. In this case it remains unclear 
whether continuity is purely technical. Narens (1985, Theorem 1.8.3) gives an example where an Archimedean axiom does add empirical meaning to other axioms.

Now we can address the contribution of Theorem 3.1. Without this theorem one might at first sight conjecture the following. Suppose that continuity with respect to topological connectedness is satisfied, so that the algebraic approach can be adopted, but, at first sight, the topological approach possibly cannot be. Then one may conjecture that the algebraic approach will lead to noncontinuous representations, so representations intrinsically different from those that result when the topological approach is adopted. Then, to apply the results derived in the topologically orientated literature for the representations obtained there, one would still have to check the remaining condition of topological separability or determine continuity in another way. Theorem 3.1 shows that the conjecture as described above does not hold. The algebraic approach does not lead to an intrinsically different representation, and one can indeed apply the results from the topologically orientated approach. An example of such results is, for the context of decision making under uncertainty (Section 6), the study of comparisons of risk aversion in the attitudes of people towards risk, as initiated by Pratt (1964) and Arrow (1971). That such results can be obtained under continuity restrictions, without the need for differentiability restrictions, has been shown in Wakker, Peters, and Van Riel (1985).

Whenever both the algebraic and the topological approach can be used, they lead to the same representation, Thus, by Adams, Fagot, and Robinson (1970; analogous to their Theorem 3) at least part of the nonempirical implications of continuity has been identified by the algebraic approach: that contained in the Archimedean axiom.

Another question concerns the appeal of the conditions. How close are the algebraic and topological conditions to an intuitive perception of human beings? We are not aware of actual experiments to determine this, so let us only give a personal opinion and some references. We find continuity an appealing condition when formulated in a space with a natural metric. The condition of topological connectedness is less easy to appreciate. Solvability in our opinion is more directly related to intuition and preferences than to continuity with respect to a topologically connected space. Let us finally refer to authors arguing for continuity in several contexts, where one should realize that continuity has a much longer history. Savage (1954, p. 77, in particular line 16) argues for the naturalness and usefulness of using infinite models with continuity. Arrow (1971, p. 48, lines 25 on), in a diffcrent context with a Monotone Continuity condition, writes: "The assumption of Monotone Continuity seems, I believe correctly, to be the harmless simplification almost inevitable in the formalization of any real-life problem." Richter (1980), for the context of ordinal measurement, argues for the use of general topology, rather than the less general metrical spaces, or the still less general Euclidean spaces. Our paper, for the context of additive representations, has argued for the still more general algebraic approach. 


\section{CONCLUSION}

This paper has studied additive representing functions and their specified forms. We have shown how results, obtained by the algebraic approach of KLST, can be applied to topological contexts. We have argued that the algebraic approach is preferable to the topological approach.

\section{REFERENCES}

Adams, E. W., \& FaGOT, R. F. (1959). A model of riskless choice. Behavioral Science, 4, 1-10.

Adams, E. W., FaGot, R. F., \& Robinson, R. (1970). On the empirical status of axioms in theories of fundamental measurement. Journal of Mathematical Psychology, 7, 379-409.

Alt, F. (1936). Ueber die Messbarkeit des Nutzens. Zeitschrift für Nationalökonomie, 7, 161-169; translated into English by SсHACH, S. (1971). On the measurability of utility. In J. S. Chipman, L. Hurwicz, M. K. Richter, \& H. F. Sonnenschein (Eds.), Preferences, utility, and demand (Chap. 20). New York: Harcourt Brace Jovanovich.

ARrow, K. J. (1971). Essays in the theory of risk-bearing. Amsterdam: North-Holland.

BASU, K. (1982). Determinateness of the utility function: Revisiting a controversy of the thirties. Review of Economic Studies, 49, 307-311.

BOWEN, R. (1968). A new proof of a theorem in utility theory. International Economic Review, 9, 374.

CoOmbs, C. A., Bezembinder, Th. G. G.. \& GoOde, F. M. (1967). Testing expectation theories without measuring utility or subjective probability. Journal of Mathematical Psychology, 4, 72-103.

COOTER, R. D., \& RAPPOPORT, P. (1984). Were the ordinalists wrong about welfare economics? Journal of Economic Literature, 22, 507-530.

COOTER, R. D., \& RAPPOPORT, P. (1985). Reply to I. M. D. Little's comment. Journal of Economic Literature, 23, 1189-1191, 367-373.

Debreu. G. (1954). Representation of a preference ordering by a numerical function in R. M. Thrall, C. H. Coombs, \& R. L. Davis (Eds.), Decision processes (pp. 159-165). New York: Wiley.

Derreu, G. (1960). Topological methods in cardinal utility theory. In K. J. Arrow, S. Karlin, \& P. Suppes (Eds., 1959), Mathematial methods in the social sciences (pp. 16-26). Stanford: Stanford Univ. Press.

Debreu, G. (1964). Continuity properties of paretian utility. International Economic Review, 5, 285-293.

FalmaGne, J. C. (1976). Random conjoint measurement and loudness summation. Psychological Review, $83,65-79$.

FrenCH, S., \& Vassiloglou, M. (1986). Strength of performance and examination assessment. British Journal of Mathematical Psychology, 39, 1-14.

FUhrKen, G., \& Richter, M. K. (1988). Algebraic methods in cardinal utility theory. In W. Eichhorn (Ed.), Measurement in economics (Theory and Applications of Economic Indices) (pp. 311-326), Heidelberg: Physica-Verlag.

GoRman, W. M. (1968). The structure of utility functions. Revien of Economic Studies, 35, 367-390.

Gorman, W. M. (1971). Apologia for a lemma. Review of Economic Studies 38, 114; Clontarf revisited. Review of Economic Studies, 38, 116.

Grodal. B. (1978). Some further results on integral representation of utility functions. Institute of Economics, University of Copenhagen, Copenhagen.

Jafrray, J.-Y. (1974a). Existence, Propriétés de Continuité, Additivité de Fonctions dutilité sur un Espace Partiellement ou Totalement Ordonné. Ph.D. dissertation, Université de Paris VI, Paris.

JAFFRAY, J.-Y. (1974b). On the extension of additve utilities to infinite sets. Journal of Mathematical Psychology, 11, 431-452.

JAFFRAY, J.-Y. (1975). Existence of a continuous utility function: An elementary proof. Econometrica, 43, 981-983.

KeLLEY, J. L. (1955). General topology. London: Van Nostrand.

Koopmans, T. C. (1972). Representations of preference orderings with independent components of 
consumption; Representations of preference orderings over time. In C. B. McGuire \& Radner (Eds.), Decision and organization (pp. 57-100). Amsterdam: North-Holland.

Krantz, D. H., Luce, R. D., Suppes, P. \& Tversky, A. (KLST) (1971). Foundations of measurement. Vol. I. Additive and polynomial representations. New York: Academic Press.

Little, I. M. D. (1985). Robert Cooter and Peter Rappoport, "Were the ordinalists wrong about welfare economics?": A comment. Journal of Economic Literature, 23, 1186-1188.

LuKas, J. (1987). Additiv Verbundene Messung der Wahrgenommenen Flächengrösse: Ein Experimentelles Verfahren zur Lösung des Testbarkeitsproblems. Zeitschrift für Experimentelle und Angewandte Psychologie, 34, 416 430.

LUCE, R. D. (1966). Two extensions of conjoint measurement. Journal of Mathematical Psychology, 3, 348-370.

MURPHY, F. P. (1981). A note on weak separability. Review of Economic Studies, 48, 671-672.

NARENS, L. (1985). Abstract measurement theory. London; MIT.

PRATT, J. W. (1964). Risk aversion in the small and in the large. Econometrica, 32, 122-136.

RICHTER, M. K. (1980). Continuous and semi-continuous utility. International Economic Review, 21, 293-299.

RosKAM, E. E. (1987). ORDMET3: An improved algorithm to find the maximin solution to a system of linear (in )equalities. Report 87MA06, Universily of Nijmegen, Department of Mathematical Psychology.

SAVAGE, L. J. (1954). The foundations of statistics. New York: Wiley; (2nd ed., 1972). New York: Dover.

SсотT, D. (1964). Measurement structures and linear inequalities. Journal of Mathematical Psychology, 1, 233-247.

Sertel, M. R. (1972). A four-flagged lemma. Review of Economic Studies, 39, 487-490.

SHAPLEY, L. S. (1975). Cardinal utility comparisons from intensity comparisons. Report R-1683-PR. The Rand Corp., Santa Monica, CA.

SHubIK, M. (1982). Game theory in the social sciences. Cambridge: MIT Press.

TitiEv, R. J. (1969). Some model-theoretic results in measurement theory. Technical Report No. 146, Psychology Series, Institute for Mathematical Studies in the Social Sciences, Stanford University.

Tversky, A. (1964). Finite additive structures. Michigan Mathematical Psychology Program, MMPP 64-6, University of Michigan.

TVERSKY, A. (1967). Additivity, utility, and subjective probability. Journal of Mathematical Psychology, 4, 175-201.

TVErSKy, A. (1977). Features of similarity. Psychological Review, 84, 327-352.

TVERSKY, A., \& KRANTZ, D. H. (1969). Similarity of schematic faces: A test of interdimensional additivity. Perception and Psychophysics, 5, 124-128.

VIND, K. (1971). The structure of utility functions. Review of Economic Studies, 38, 113; Comment. Review of Economic Studies, 38, 115.

Vind, K. (1974). A Note on a four-flagged lemma. Review of Economic Studies, 41, 571.

WAKKER, P. P. (1984a). Cardinal coordinate independence for expected utility. Journal of Mathematical Psychology, 28, 110-117.

WAKKER, P. P. (1984b). Continuous expected utility for arbitrary state spaces. Methods of Operations Research, 50, 113-129.

Wakker, P. P., Peters, H. J. M., \& Van Riel, T. B. P. L. (1985). Comparisons of risk aversion, with an application to bargaining. Methods of Operations Research, 54, 307-320.

WAKKER, P. P. (1986). Concave additively decomposable representing functions and risk aversion. In L. Daboni, A. Montesano, \& M. Lines (Eds.). Recent developments in the foundations of utility and risk theory (pp. 249-262). Dordrecht: Reidel.

WAKKER, P. P. (1988). Characterizations of quasilinear representing functions, and specified forms of these. In W. Eichhorn (Ed.), Measurement in economics (Theory and Applications of Economic Indices) (pp. 311-26). Heidelberg: Physica-Verlag.

ReCeIVED: July 27, 1987 